\title{
Flow cell to study crystallisation processes in-situ using terahertz time-domain spectroscopy
}

\author{
Qi Li*, Johanna Kölbel*, Terry Threlfall, and J. Axel Zeitler
}

\begin{abstract}
A versatile setup based on terahertz time-domain spectroscopy was developed to monitor the process of crystallisation and characterise the structure of the crystals as well as the dynamics of the solvent in situ. The setup can be used to investigate crystallisation processes over a wide range of temperature, either under static conditions or during continuous flow. An attached optical imaging probe can be used to monitor the process in parallel to the terahertz spectroscopy investigation. The implementation of terahertz spectroscopy offers a great opportunity to investigate the dielectric relaxation dynamics of the solvent and the vibrational modes characteristic for the crystalline phases simultaneously. The details of the crystallisation setup are demonstrated at the example of the crystallisation process of magnesium sulphate heptahydrate from its aqueous phase.
\end{abstract} istry

Index Terms-THz-TDS, crystallisation, microfluidics, chem-

\section{INTRODUCTION}

C RYSTALLISATION has been an intriguing topic in both crystallography and chemistry for decades, yet resolving its fundamental nature at the molecular level is still not straightforward. This is due to the complexity of the seemingly simple process which can give the impression of a highly erratic process. Nucleation and crystal growth are widely understood to constitute the two main steps [1], [2].

A number of techniques have been applied to study the crystallisation process. X-ray techniques, such as small-angle and wide-angle $\mathrm{X}$-ray scattering, X-ray spectroscopy, and Xray diffraction, are of great importance in providing information on the molecular level. In addition, spectroscopic methods can be used to probe the chemical interactions in the system of interest, including NMR spectroscopy, FTIR, Raman, and UV/Vis spectroscopy [2]. Typically, a combination of selected techniques is necessary to fully characterise a system, and in recent years computational methods haven been used increasingly to great effect to complement the experimental investigations.

In aqueous systems, the high absorption of water in the infrared results in a noisy background signal, which complicates FTIR measurements and limits their application largely

Q. Li, J. Kölbel, and J.A. Zeitler are with the Department of Chemical Engineering and Biotechnology, University of Cambridge, Cambridge, UK e-mail: jaz22@cam.ac.uk.

T. Threlfall is with the Department of Chemistry, University of Southampton, Southampton, UK.

*The first two authors contributed equally to this work

QL would like to thank the Chinese Scholarship Council for funding. JK would like to thank the EPSRC Cambridge Centre for Doctoral Training in Sensor Technologies and Applications (EP/L015889/1) and AstraZeneca for funding. to measurements near surfaces in attenuated total reflectance (ATR). Raman spectroscopy is therefore commonly used as an alternative to investigate the vibrational modes in the infrared given the lack of contribution from water molecules to the Raman spectra due to the different selection rules. In recent years, terahertz time-domain spectroscopy (THz-TDS), has emerged as a new tool that can be used, similar to FTIR, to probe the molecular vibrations due to permanent dipole changes but it has the advantage that it can also be applied in transmission when water is present in the sample given the slightly lower absorption of water at terahertz frequencies compared with the mid-infrared. THz-TDS has been proved to be a useful technique to study both amorphous and crystalline materials in the solid state, as it is sensitive to inter- and intra-molecular interactions within such systems [3]. It has been successfully applied to investigate the properties of pure amorphous materials and crystals, and also phase transitions of polymorphs and even crystallisation from the amorphous to crystalline phase [3]-[5]. Additionally, the dielectric relaxation phenomena on picosecond time scales have been investigated with terahertz spectroscopy in polar liquids, such as alcohol, water, and their mixtures [6].

However, there are not yet many studies utilising THz-TDS to investigate the crystallisation process in solutions. May and Taday employed terahertz spectroscopy in ATR configuration and monitored the crystallisation of sucrose in confectionary products [7]. The results clearly showed the transition from sugar solution to a solid glassy and then crystalline state. Soltani et al. refined this method and successfully proved the existence of intermediate hydrate states in the crystallisation of L-(+)-tartaric acid during the evaporation of water from aqueous solutions of tartaric acid solutions [8]. Terahertz spectra were acquired over a period of $60 \mathrm{~h}$ while water evaporated and three stages of crystallisation were discernible. At the beginning, the spectra of the solution phase were featureless. In the nucleation stage, the absorption decreased as a result of the reduction in water concentration due to evaporation. In some experiments discontinuous increases in absorption were observed which were attributed to water being released when the hydrated states transformed to the final crystals. A feature was spotted at approximately $750 \mathrm{GHz}$ that was hypothesised to result from the vibration of water molecules surrounding the fragments of solvent molecules. The final observed stage was the crystalline phase displaying a dominant absorption feature at $1.1 \mathrm{THz}$. These three stages were assumed to be happening inhomogeneously throughout the probed volume.

Even though the absorption of terahertz radiation from water is approximately two orders of magnitude weaker than in 
the mid-infrared it is still around $200 \mathrm{~cm}^{-1}$ at $1 \mathrm{THz}$, which limits the path length of the cuvette that can be used for transmission measurements to around $100 \mu \mathrm{m}$ in terms of trading off dynamic range and spectral bandwidth for the measurement. However, such a path length is sufficient for crystallisation experiments and terahertz spectroscopy combined with microfluidics offers the opportunity to measure samples containing water with high sensitivity and over a wide frequency range. Similar approaches using thin cuvettes in transmission were previously found to be useful to investigate biomaterials such as proteins and DNA [9], [10].

In the newly developed set up, a closed microfluidics platform made of z-cut quartz was utilised which enables investigation of both the solvent and solute at frequencies from $0.35 \mathrm{THz}$ to $2 \mathrm{THz}$ whilst allowing for optical observations in the visible range of the spectrum during crystallisation and for cleaning of the cell between experiments. Magnesium sulphate hydrates were chosen as a model system, as different hydrates are known to form under specific conditions. Between $2{ }^{\circ} \mathrm{C}$ to $47^{\circ} \mathrm{C}, \mathrm{MgSO}_{4} \cdot 7 \mathrm{H}_{2} \mathrm{O}$ is the predominant form that can be crystallised at reasonable experimental time scales [11]. Therefore, $\mathrm{MgSO}_{4} \cdot 7 \mathrm{H}_{2} \mathrm{O}$ is used as an example to demonstrate the setup designed based on transmission $\mathrm{THz}-$ TDS that can observe the crystallisation and characterise the structure before, during, and after crystal formation in situ.

\section{METHODS}

Up to now, all published studies using terahertz spectroscopy to study crystallisation from solution have relied on solvent evaporation to trigger nucleation. These studies were performed by ATR sampling, which restricts the observations to the evanescent field near the surface of the ATR crystal rather than in the bulk of the liquid. The goal of this project was hence to build a versatile setup that would be capable to study crystallisation mechanisms from supersaturated solutions with THz-TDS in transmission geometry. Main design criteria were intended to cover operation over a wide of temperature and concentration, assure stability at high temperature, and control temperature gradients during crystallisation, as well as being able to measure under stagnant and flowing conditions.

\section{A. Description of the setup}

The setup comprised a temperature controlled crystallisation cell that was designed such that it fits into a standard transmission chamber of a commercial THz-TDS setup (Figure 1). In parallel to the terahertz measurements it was possible to film the crystallisation process using a small camera that was integrated into the setup.

The central part of the setup was the crystallisation cell that held a cuvette containing the solution during the measurements. The cuvette was manufactured by Hellma (Southendon-Sea, UK). It was made of two pieces of z-cut quartz (each $1.5 \mathrm{~mm}$ thick to avoid etalon reflections) glued together using EP220 epoxy (Fiberdur, Aldenhoven, Germany) with an outlet on each side giving a pathway of solution. The channel was

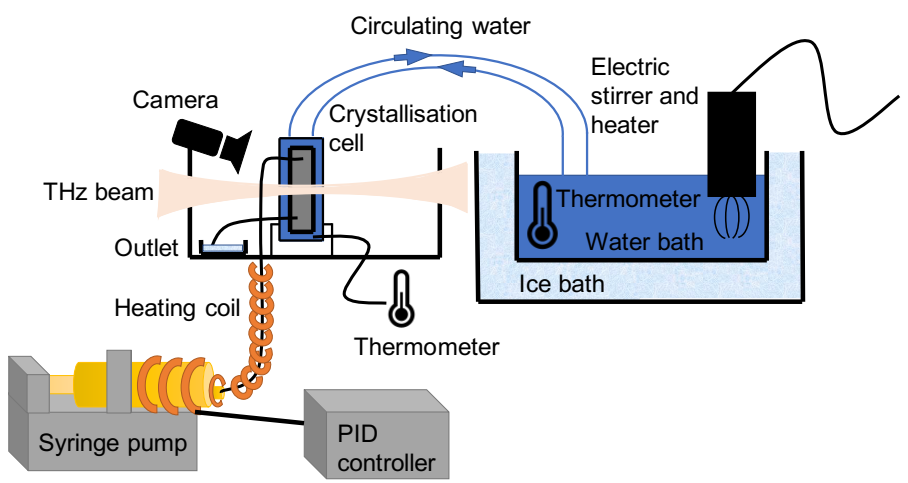

Fig. 1. Schematic setup.
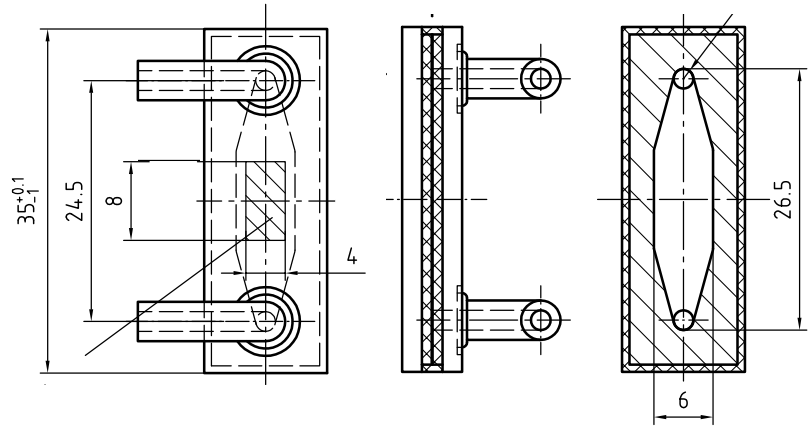

Fig. 2. Three-view drawing of the cuvette. Left: view from the front, centre: view from the side, right: view from the bottom. Lengths are in $\mathrm{mm}$.

$24.5 \mathrm{~mm}$ long and $100 \mu \mathrm{m}$ thick, and a schematic drawing is shown in Figure 2.

The quartz cuvette was held in a metal jacket which has been machined with channels to allow for water to circulate through the metal block in order to control the temperature of the quartz cuvette using a water bath and pump (Figure 3). A thermocouple was fitted to the block to allow for accurate temperature control. During the THz-TDS measurement, the assembled crystallisation cell was positioned at the focal position of the measurement chamber and the terahertz beam propagated through the centre of the cuvette with a beam width of about $2 \mathrm{~mm}$.

The temperature of the crystallisation cell was controlled by an external recirculating water bath that was connected to the inlet and outlet of the sample holder metal jacket.
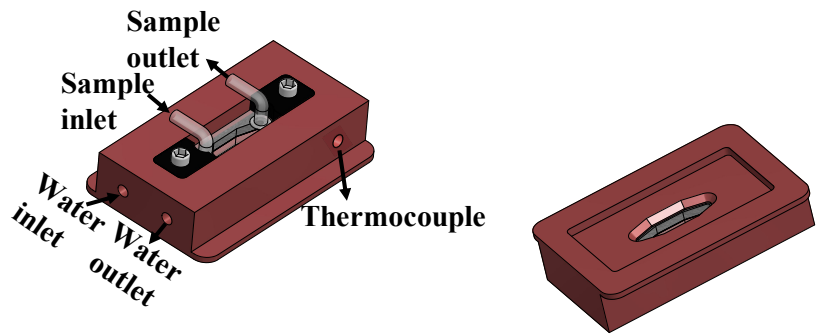

Fig. 3. Renderings of the metal sample holder with cuvette inserted. 
The pipes between water bath and crystallisation cell were insulated using foam pipe lagging, circulation of the coolant/ heating medium was performed using a pump (120s cased pump, Watson Marlow, Falmouth, UK), and the temperature was controlled by balancing variable heating with an in-built stirrer and PID controller (Anova Applied Electronics, San Francisco, CA, USA) against the near constant cooling from a surrounding ice bath.

Using this setup it was possible to vary the temperature setpoint by $0.1^{\circ} \mathrm{C}$ intervals and it was possible to ramp the temperature over the course of a measurement. Using water and ice as the respective circulating and cooling media, the temperature of the crystallisation cell up could be equilibrated between $4{ }^{\circ} \mathrm{C}$ to $90{ }^{\circ} \mathrm{C}$ without further adjustments. Crystallisation was triggered by slowly decreasing the temperature of the crystallisation cell.

In order to investigate the effect of continuous sample flow, a syringe pump (Aladdin Syringe ONE Programmable Syringe Pump AL-1000, World Precision Instruments, Hitchin, UK) was used to provide a steady flow of solution. At its lowest setting, the flow was $0.375 \mathrm{~mL} \mathrm{~min}^{-1}$, which corresponded to a residence time of approximately $2.4 \mathrm{~s}$ within the sample cuvette when assuming plug flow. The syringe was contained by a metal tube which was wrapped with a heating coil (SRT051040LSE, silicon rubber tape, Omega Engineering, Manchester, UK). This ensured that the heat provided by the heating coil was evenly distributed and single-use syringes could easily be replaced between measurements. Further heating coil was wrapped around the transfer tube all the way to the inlet of the crystallisation cell. This made it possible to control, and keep the temperature constant both inside the syringe and the transfer tube.

The temperature at the syringe was measured with a thermocouple and controlled via a PID controller (P6100, West Control Solutions, Brighton, UK). The syringe was kept at an elevated temperature to avoid supersaturation and to minimise the chance of nucleation and crystallisation occurring in the solution before reaching the cell. Over the course of the experiment, the solution was pumped into the crystallisation cell. The temperature at the inlet of the cell was again measured with a thermocouple to assure that the temperature was still high enough to avoid nucleation before the solution reaches the cell. This thermocouple was not connected to the PID controller and has been added only to monitor the system. Once the solution had passed through the cell, it flowed through the outlet at the bottom side to a beaker that was placed within the sample compartment of the terahertz spectrometer to collect the solution that was purged out.

An optical camera (CF-25, CrazyFire, China) was attached to the cell and focused on the liquid layer in order to acquire images every $2 \mathrm{~s}$ of the crystallisation process in addition to the terahertz spectra.

The temperature was monitored continuously throughout the crystallisation experiments using thermocouples located in the water bath, at the heater, and inside the flow cell, as well as at the inlet as mentioned above. Temperature data was acquired once every second.

\section{B. Sample preparation and operation}

The $\mathrm{MgSO}_{4}$ water solution was prepared by dissolving $\mathrm{MgSO}_{4} \cdot 7 \mathrm{H}_{2} \mathrm{O}$ (Sigma-Aldrich, Gillingham, UK) in MilliQ water (IQ 7000, Merck, Darmstadt, Germany, resistivity $18.2 \mathrm{M} \Omega \mathrm{cm}$ ) to the desired concentration in a beaker. The mixture was placed on a magnetic stirrer for constant stirring until the sample was fully dissolved. It was sometimes heated to a slightly higher temperature (in the range of $25^{\circ} \mathrm{C}$ to $35^{\circ} \mathrm{C}$ ) when preparing solutions with high concentrations to facilitate dissolution. EDTA solution was used for cleaning and purging the crystallisation cell after each measurement. For the cleaning solution, commercial EDTA solution $(\mathrm{pH}=8$; Fisher Scientific, Loughborough, UK) was mixed with $\mathrm{NaOH}$ solution (Reagecon Diagnostics, Shannon, Ireland) to adjust the $\mathrm{pH}$ to 10 , in which $\mathrm{MgSO}_{4} \cdot 7 \mathrm{H}_{2} \mathrm{O}$ has a better solubility.

The temperature of the metal jacket around the crystallisation cell was equilibrated before injecting the solution. Once the sample was injected into the crystallisation cell the temperature was either kept stable and crystallisation was observed as a function of time or it was varied using the heater control in order to induce crystallisation or dissolution.

For static measurements, solution was injected outside the spectrometer to avoid spillage inside the sample chamber. Tubes were connected to the inlet and outlet of the cell and solution was injected with a syringe from one end. It was taken care that no air bubbles remained within the crystallisation cell. Surplus solution purged from the cell was collected in a beaker and disposed of.

Previously it had been observed that crystals tend to form either at the inlet or outlet of the cell, or, if connected, at the end of tubes. To ensure higher reproducibility, the inlet and outlet tubes were truncated at a length of $3 \mathrm{~cm}$ and the ends sealed with parafilm to avoid evaporation of the sample. It would also have been possible to do without tubes, however, due to the small size of the crystallisation cell and the need to seal the inlet and outlet, this often introduced air bubbles so that it was decided to use tubes of a constant length instead.

Following injection, the sample holder was immediately placed into the chamber and measurement acquisition was initiated no longer than $30 \mathrm{~s}$ after injecting the sample.

For the continuous flow measurements, a suitably large syringe was first fully filled with the prepared $\mathrm{MgSO}_{4}$ solution, then placed into the metal tube that was kept at the set-point temperature. The syringe within the temperature controlled metal jacket was then fixed to the syringe pump and connected to the inlet tube before starting the measurements.

In either case, static or continuous flow, once the sample was injected into the crystallisation cell the temperature was either kept stable or varied using the circulating water bath, depending on the type of experiment performed.

To clean the flow cell and to remove remaining crystals after an experiment was completed, the cell was purged with the cleaning solution several times before and after each measurement, additionally with Milli-Q water as well. The spectrometer was used to confirm the absence of crystals after purging. 


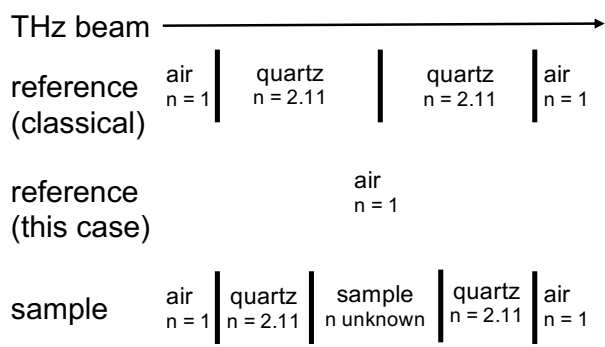

Fig. 4. Schematic of the path of the terahertz beam in the sample chamber for different cases (quartz windows as reference, air as reference, and the sample sandwiched between windows). $n$ denotes the real part of the refractive index.

\section{Terahertz time-domain spectroscopy}

A TeraPulse 4000 (Teraview, Cambridge, UK) spectrometer was used to perform the terahertz transmission measurements. The thickness of the z-cut quartz windows comprising the flow cell, as well as the need to use air as the reference, caused a relatively large optical delay between the reference pulse and the sample pulse for these measurements. Therefore, the high-resolution mode was chosen, which allowed for acquiring a time-domain waveform of 45 ps duration.

Each spectrum was calculated from the co-average of 15 individual waveforms, resulting in the acquisition of one spectrum per $20 \mathrm{~s}$. The spectral resolution set in the software was $0.94 \mathrm{~cm}^{-1}$. Given the dynamic range of the spectrometer and the absorption of water in the terahertz region, high quality spectroscopic data were acquired in the frequency range of $0.3 \mathrm{THz}$ to $2 \mathrm{THz}$.

\section{RESULTS AND DISCUSSION}

\section{A. Data processing}

During a THz-TDS measurement, the waveforms of reference and sample were recorded. The ratio of the Fourier transforms of reference and sample (the so-called power spectrum) yielded the complex transmission coefficient of the sample as a function of frequency.

In their paper from 1996 Duvillaret et al. described a reliable method to extract optical constants from THz-TDS measurements by taking the complex refractive indices of the surrounding media and sample into consideration, and solving the inverse problem of extracting the complex index of refraction iteratively from the measured transmission coefficient [12].

In this case however, the analysis was complicated by the fact that the surrounding media were not the same for the reference and sample measurement, as is demonstrated in Figure 4. To avoid the Fabry-Pérot effect from internal reflections caused by the empty crystallisation cell, the reference measurements were performed in air without quartz windows.

This posed a challenge when attempting to calculate the complex optical constants using the method of Duvillaret et al. because their method assumed the surrounding media for the reference and sample measurement were the same.

The absorption of z-cut quartz windows in the terahertz region of interest is very low, with an extinction coefficient
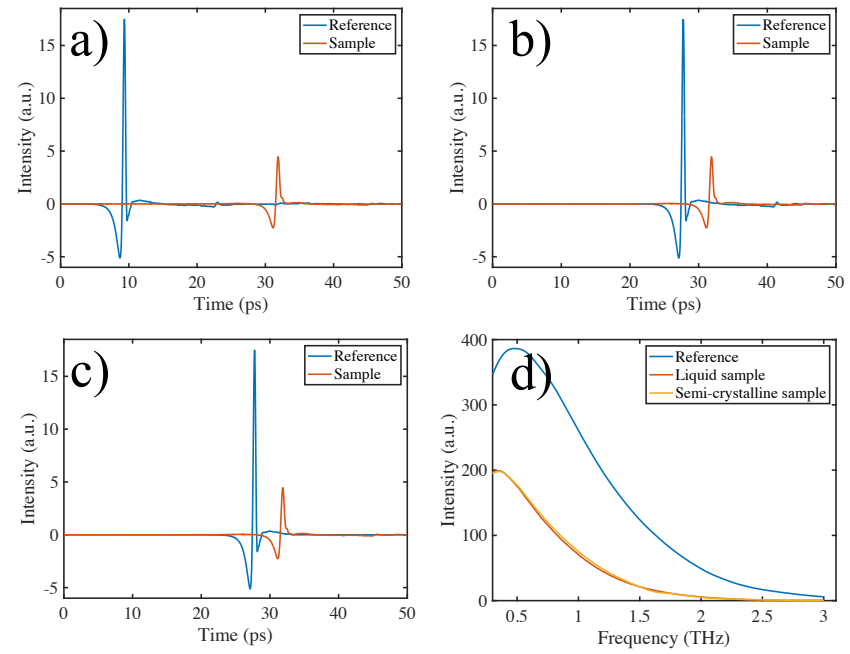

Fig. 5. a)-c): Reference (blue) and sample (orange) waveforms. a): original case, b): after shifting the reference waveform, c): after shifting and zeropadding the reference waveform. d) Power spectra of reference (blue) and sample (red and yellow). In red, a liquid sample and in yellow after crystallisation.

of approximately zero and the real part of the refractive index being approximately constant. Inserting windows into the beam path mostly lead to a longer time delay before detecting the pulse on the order of $20 \mathrm{ps}$ for a window thickness of $3 \mathrm{~mm}$. This could be utilised when calculating the absorption coefficient. While the spectral shape of the absorption was not influenced by the reference thickness, the absolute values were. By shifting the acquired reference waveform by about $20 \mathrm{ps}$ and subsequently using the method of Duvillaret et al., the absorption coefficient was calculated. The absolute shift necessary depended on the thickness of the windows and sample. By comparing the absorption coefficient of pure water with literature values, introducing a time delay of $18.43 \mathrm{ps}$ to the reference data recovered the absorption. This is illustrated in Figure 5a and $5 b$.

Due to the optical design of the Terapulse 4000 instrument, a small feature was visible in the reference waveform at a time-delay of approximately 15 ps following the main peak. This introduced artefacts in the frequency domain unless temporal windowing was applied. The reference waveform was therefore zero-padded to remove those artefacts and Figure $5 \mathrm{c}$ shows both the reference and sample waveforms that were being used to calculate the absorption coefficient. Application of a Fourier transformation resulted in the power spectra shown in Figure 5d.

The different sets of data (time, THz-TDS, temperature, images) had to be linked together prior to further analysis. Temperature data was acquired each second, an image every 2 seconds, and THz-TDS data every 20 seconds. The temperature assigned to each terahertz spectrum was calculated as the average temperature during its acquisition. This way, terahertz data could be plotted as a function of time as well as temperature and linked to images via their time stamps for visual clarification.

Whilst the molar absorptivity $\left(\mathrm{L} \mathrm{mol}^{-1} \mathrm{~cm}^{-1}\right)$ is commonly 


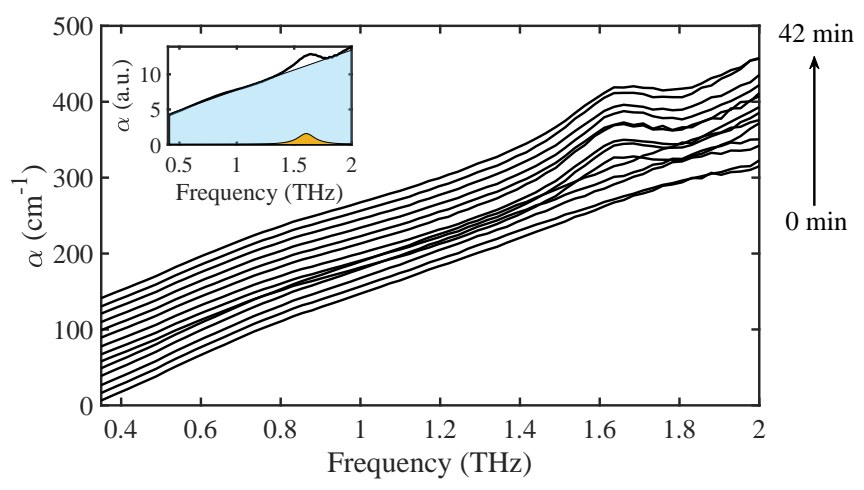

Fig. 6. Spectra recorded at different times throughout the process. Each subsequent spectrum is offset by $10 \mathrm{~cm}^{-1}$. Inset: Typical spectrum of the crystalline system. It is depicted here as the sum of the amorphous background (blue) and the peak at $1.6 \mathrm{THz}$ (orange).

used as a unit of absorption intensity in chemistry we have continued the analysis in keeping with common practice in the terahertz literature in terms of the absorption coefficient $\left(\mathrm{cm}^{-1}\right)$ for consistency.

\section{B. Results and discussion}

When operated under stagnant condition the concentration of $\mathrm{MgSO}_{4}$ in the solution within the cuvette was constant and crystallisation could be observed as a function of time, depending on the chosen temperature and concentration. At concentrations of $25 \mathrm{wt} \%$ to $29 \mathrm{wt} \% \mathrm{MgSO}_{4}$ crystallised into its heptahydrate form within minutes at temperatures between $4{ }^{\circ} \mathrm{C}$ to $12{ }^{\circ} \mathrm{C}$.

In Figure 6, the crystallisation of a supersaturated $\mathrm{MgSO}_{4}$ solution over time is shown while the sample was being kept at approximately $4{ }^{\circ} \mathrm{C}$. As expected, the spectrum of the $\mathrm{MgSO}_{4}$ solution was completely featureless with a monotonously increasing baseline. Such spectral features are expected for aqueous solutions where no molecular order on the timescales of picoseconds is maintained and thus the molecules in solution are completely disordered in an amorphous phase. Over time the baseline dropped, and a peak emerged at $1.6 \mathrm{THz}$ which showed the existence of crystals.

Magnesium sulfate heptahydrate - water spectra containing crystalline features could hence be separated into the sum of the amorphous background and the peak at $1.6 \mathrm{THz}$, as shown in the inset in Figure 6a.

\section{CONCLUSions}

We developed a setup that can be used to observe crystallisation processes from aqueous and other organic solvents utilising terahertz time-domain spectroscopy in transmission geometry. Both static systems and systems under flow were investigated while either keeping the temperature constant or employing temperature ramping. Utilising the versatility of the setup, the system was studied at different temperatures before, during, and after crystals formed, thereby allowing the investigation of both crystals and the solvent at different levels of saturation.
The effects of temperature and concentration changes will be investigated further. The setup is designed for operating temperatures between $4{ }^{\circ} \mathrm{C}$ to $90^{\circ} \mathrm{C}$, which can be extended with minor adjustments. Therefore, it can be applied to a wide range of crystalline systems at various concentrations. Future work will focus on being able to trigger crystallisation within the cell, allowing to observe the very beginning of nucleation as well as crystal growth.

\section{REFERENCES}

[1] J. Hulliger, "Chemistry and crystal growth," Angewandte Chemie International Edition in English, vol. 33, no. 2, pp. 143-162, 1994.

[2] R. J. Davey, S. L. M. Schroeder, and J. H. ter Horst, "Nucleation of organic crystals-a molecular perspective," Angewandte Chemie International Edition, vol. 52, no. 8, pp. 2166-2179, 2013.

[3] J. Sibik, M. J. Sargent, M. Franklin, and J. A. Zeitler, "Crystallization and phase changes in paracetamol from the amorphous solid to the liquid phase," Molecular Pharmaceutics, vol. 11, no. 4, pp. 1326-1334, 2014.

[4] J. A. Zeitler, D. A. Newnham, P. F. Taday, T. L. Threlfall, R. W. Lancaster, R. W. Berg, C. J. Strachan, M. Pepper, K. C. Gordon, and T. Rades, "Characterization of Temperature-Induced Phase Transitions in Five Polymorphic Forms of Sulfathiazole by Terahertz Pulsed Spectroscopy and Differential Scanning Calorimetry," Journal of Pharmaceutical Sciences, vol. 95, no. 11, pp. 2486-2498, Nov. 2006.

[5] E. P. Parrott and J. A. Zeitler, "Terahertz time-domain and low-frequency raman spectroscopy of organic materials," Appl. Spectrosc., vol. 69, no. 1, pp. 1-25, Jan 2015.

[6] R. Li, C. D’Agostino, J. McGregor, M. D. Mantle, J. A. Zeitler, and L. F. Gladden, "Mesoscopic structuring and dynamics of alcohol/water solutions probed by terahertz time-domain spectroscopy and pulsed field gradient nuclear magnetic resonance," The Journal of Physical Chemistry B, vol. 118, no. 34, pp. 10156-10 166, 2014.

[7] R. May and P. F. Taday, "Crystallization of sucrose monitored by terahertz pulsed spectroscopy," in 2013 38th International Conference on Infrared, Millimeter, and Terahertz Waves (IRMMW-THz). IEEE, 2013, pp. 1-1.

[8] A. Soltani, D. Gebauer, L. Duscheck, B. M. Fischer, H. Cölfen, and M. Koch, "Crystallization caught in the act with terahertz spectroscopy: Non-classical pathway for 1-(+)-tartaric acid," Chemistry, vol. 23, no. 57, p. 14128-14132, 2017.

[9] S. Alfihed, J. F. Holzman, and I. G. Foulds, "Developments in the integration and application of terahertz spectroscopy with microfluidics," Biosensors and Bioelectronics, p. 112393, 2020.

[10] M. Nagel, P. Haring Boilvar, M. Brucherseifer, H. Kurz, A. Bosserhoff, and R. Büttner, "Integrated thz technology for label-free genetic diagnostics," Applied Physics Letters, vol. 80, no. 1, pp. 154-156, 2002.

[11] T. M. Duncan and J. A. Reimer, Chemical Engineering Design and Analysis: An Introduction. Cambridge: Cambridge University Press, 2019.

[12] L. Duvillaret, F. Garet, and J.-L. Coutaz, "A reliable method for extraction of material parameters in terahertz time-domain spectroscopy," IEEE Journal of selected topics in quantum electronics, vol. 2, no. 3 , pp. 739-746, 1996.

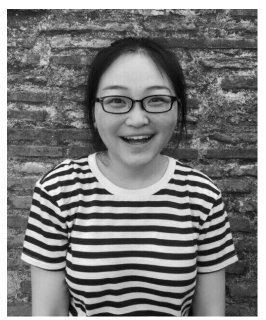

Qi Li received her B.Eng. degree in chemical engineering from the University of Edinburgh, UK and Dalian University of Technology, China, in 2017. In the same year, she joined the Terahertz Applications Group at the University of Cambridge as a $\mathrm{Ph} . \mathrm{D}$. candidate under the supervision of Prof. Axel Zeitler. Her research applies terahertz time-domain spectroscopy (THz-TDS) and density functional theory (DFT) simulations to investigate the molecular dynamics and the properties of crystalline systems. 


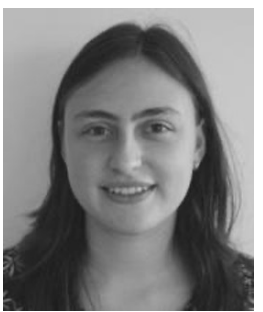

Johanna Kölbel received a B.Sc.(2017) in physics from Friedrich Schiller University Jena, Germany and a M.Res. (2018) in Sensor technologies and applications from Cambridge University, UK. Since then, she has been working on terahertz spectroscopy of liquids and amorphous solids during her $\mathrm{PhD}$, supervised by Prof. Axel Zeitler in the Terahertz Applications group at the University of Cambridge.



Terry Threlfall has degrees in chemistry and law and a Ph.D. in organic chemistry, all from London University. After doing post-doctoral work with Prof. Eschenmoser in Zurich, he worked for 30 years in industry, mainly in the pharmaceutical industry and then for 30 years in academia, firstly at the University of York then at the University of Southampton. His interests are in infrared spectroscopy, polymorphism and crystallisation.

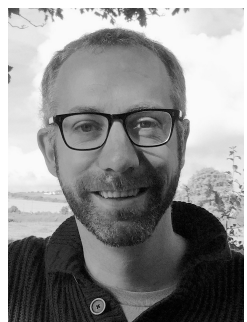

J. Axel Zeitler received the Staatsexamen from Würzburg, Germany, in 2002, the PGCert degree and the Ph.D. degree from the University of Otago, Dunedin, New Zealand, in 2003 and 2007, respectively. He holds the Chair of Microstructure Engineering at the Department of Chemical Engineering and Biotechnology at University of Cambridge where he leads the Terahertz Application Group focusing on terahertz spectroscopy and imaging for material characterisation as well as non-destructive imaging supported by complementary techniques. 